\title{
Contribuição do aproveitamento integral dos alimentos para saúde e meio ambiente
}

\begin{abstract}
Atualmente, são desperdiçados no mundo cerca de 1,3 bilhões de toneladas de alimentos. O Brasil é considerado o quarto maior produtor de alimento do mundo e ao mesmo tempo, está entre os dez países que mais desperdiçam. A preocupação com o desperdício e também o esgotamento dos recursos naturais, exige de todos, novos comportamentos e novas formas de pensar e agir. Para que esse cenário mude, a maneira mais indicada é praticar o aproveitamento integral dos alimentos, utilizando as partes não convencionais como a casca, talo, folha e semente. Esse, poderá contribuir para a redução da produção de resíduos alimentares, bem como, minimizar o impacto ambiental causado pelos mesmos e também em ações que visem reduzir a fome e desnutrição, por meio do desenvolvimento de técnicas dietéticas que reaproveitem integralmente os alimentos. Assim, o objetivo do presente trabalho foi realizar uma revisão bibliográfica da importância do aproveitamento integral dos alimentos. Para isso, foi realizada com base em artigos publicados nos últimos 10 anos, com idioma português ou inglês, além de textos na íntegra. Pode-se concluir que os alimentos quando consumidos na sua forma integral podem oferecer diversos benefícios aos consumidores, no tratamento e/ou na prevenção de doenças como diabetes, hiperlipidemias, obesidade, constipação e neoplasias, devido a maior presença de fibras, melhora do sistema imunológico, auxiliando na perda de peso corporal e aumentando a ingestão de nutrientes. É de suma importância a educação sobre as partes não convencionais dos alimentos para possibilitar a população uma alimentação mais nutritiva, segura, sustentável, prevenindo assim as doenças crônicas não transmissíveis, melhorando a saúde e a qualidade de vida. Além disso, pode contribuir com a redução dos gastos com a alimentação, desperdício dos alimentos e geração de lixo orgânico.
\end{abstract}

Palavras-chave: Desperdício de Alimentos; Saúde; Nutrição; Sustentabilidade.

\section{Contribution of the total utilization of food for health and the environment}

\begin{abstract}
Currently, around 1.3 billion tons of food are wasted in the world. Brazil is considered the fourth largest food producer in the world, and at the same time, it is among the ten countries that most waste. The concern with waste and also the depletion of natural resources, requires from everyone, new behaviors and new ways of thinking and acting. For this scenario to change, the best way is to practice the full use of food, using unconventional parts such as bark, stalk, leaf and seed. This can contribute to reducing the production of food waste, as well as minimizing the environmental impact caused by them and also in actions aimed at reducing hunger and malnutrition, through the development of dietary techniques that fully reuse food. Thus, the objective of the present work was to carry out a bibliographic review of the importance of the full use of food. For this, it was carried out based on articles published in the last 10 years, in Portuguese or English, in addition to full texts. It can be concluded that food when consumed in its entirety can offer several benefits to consumers, in the treatment and/or prevention of diseases such as diabetes, hyperlipidemia, obesity, constipation and neoplasms, due to the greater presence of fibers, improvement of the system immune system, aiding in weight loss and increasing the intake of nutrients. Education about the unconventional parts of food is of utmost importance to enable the population to have a more nutritious, safe, sustainable diet, thus preventing chronic non-communicable diseases, improving health and quality of life. In addition, it contributes to the reduction of spending on food, food waste and the generation of organic waste.
\end{abstract}

Keywords: Food Waste; Health; Nutrition; Sustainability.

Topic: Desenvolvimento, Sustentabilidade e Meio Ambiente

Reviewed anonymously in the process of blind peer.

Julia Hirano Rodrigues

Universidade Cesumar, Brasil

http://lattes.cnpq.br/6837729148549360

http://orcid.org/0000-0002-4194-824X

julia_hirano@hotmail.com

Rafaela Stinghen Garcia Sampaio (iD)

Universidade Cesumar, Brasil

http://lattes.cnpq.br/4299076933383503

http://orcid.org/0000-0001-9181-7902

rafaelaasampaio@hotmail.com

Lucas Dutra Zani da Silva Souza (iD)

Universidade Cesumar, Brasil

http://lattes.cnpq.br/1998654658664058

http://orcid.org/0000-0001-5662-5382

zanidutrass@gmail.com

d

DOI: 10.6008/CBPC2179-6858.2021.007.0029
Received: 02/07/2021

Approved: 28/07/2021

\author{
Tiago Ferrari (iD \\ Universidade Federal do Paraná, Brasil \\ http://lattes.cnpq.br/8137165993439844 \\ http://orcid.org/0000-0001-9292-2595 \\ dr.tiago.ferrari@gmail.com \\ Daniele Fernanda Felipe (iD) \\ Universidade Cesumar, Brasil \\ http://lattes.cnpq.br/6602186701933916 \\ http://orcid.org/0000-0001-9028-0728 \\ daniele.felipe@unicesumar.edu.br \\ Ariana Ferrari (iD \\ Universidade Cesumar, Brasil \\ http://lattes.cnpq.br/1718769915904474 \\ http://orcid.org/0000-0001-7843-8019 \\ ariana.ferrari@unicesumar.edu.br
}

\section{Referencing this:}

RODRIGUES, J. H.; SAMPAIO, R. S. G.; SOUZA, L. D. Z. S.; FERRARI, T.; FELIPE, D. F.. Contribuição do aproveitamento integral dos alimentos para saúde e meio ambiente. Revista Ibero Americana de Ciências Ambientais, v.12, n.7, p.314-327, 2021. DOI:

http://doi.org/10.6008/CBPC2179-6858.2021.007.0029 


\section{INTRODUÇÃO}

O índice de natalidade tem se sobressaído em reação as taxas de mortalidade, ainda que essas sejam altas, observa-se uma tendência significativa no crescente número de habitantes no mundo. Segundo dados publicados pela Organização das Nações Unidas (ONU), a população mundial, no ano de 2017, era de cerca de 7,6 bilhões de pessoas, e o Brasil situava-se na quinta posição, como país mais populoso do mundo, correspondendo a 209 milhões de pessoas. Conforme o autor, as expectativas populacionais para a década de 2050 estarão entre 9,3 e 10,6 bilhões de pessoas, visto que, a população do planeta cresce aproximadamente, 83 milhões de pessoas, por ano (SANTOS et al., 2020).

É importante salientar, que um em cada nove indivíduos no mundo (ou cerca de 805 milhões de pessoas) possuem hipossuficiência alimentar (SAATH et al., 2018). Neste interim, a alimentação e nutrição adequada são partes essenciais para a promoção e proteção da saúde, prevenindo uma série de patologias e auxiliando na qualidade de vida. É um dispositivo relacionado com os direitos humanos, sendo está embasada no acesso físico e econômico de todos os indivíduos aos alimentos e aos recursos, a qual está contemplada no artigo 25 da Declaração Universal dos Direitos Humanos de 1948, sendo que no ano de 2010, foi aprovada a Emenda Constitucional no 64, que inclui a alimentação no artigo 6o da Constituição Federal (BRASIL, 2008).

A partir desses dados observa-se algo conflitante no quesito alimentar, pois o Brasil está entre os dez principais países no tocante ao desperdício de alimentos no mundo, apesar de uma grande parcela da população viver sob a linha da pobreza (PORPINO et al., 2015; HENZ et al., 2017). O desperdício infelizmente está incorporado à cultura brasileira, sendo presente em todas as fases da cadeia de produção de alimentos, bem como, em todas as classes econômicas (SILVÉRIO et al., 2014). Estudos indicam que as famílias brasileiras desperdiçam em média 353 gramas de alimentos por dia ou 128,8 kg por ano. Em uma análise perca pita, o desperdício é de 114 gramas por dia, representando um resíduo de 41,6 kg por pessoa. Em 2017 o Brasil correspondia ao quinto país mais populoso do mundo, correspondendo a 209 milhões de pessoas (SANTOS et al., 2020).

O aumento do desperdício de alimentos e seus montantes de resíduos gerados a partir destas perdas, vem gerando um crescente reconhecimento e mobilização mundial, pela sua importância socioeconômica e ambiental negativa. Com todo esse adensamento global, ocasiona em uma diminuição de fontes e recursos não renováveis, perda da biodiversidade e mudanças climáticas. Mas esse problema, pode ser revertido, com a redução do desperdício gerado em todo o mundo (FILIMONAU et al., 2020; SANTOS et al., 2020).

É de extrema importância, o aproveitamento do alimento em sua integralidade, ou seja, o uso de todas as partes comestíveis dos alimentos como fonte alimentar e nutricional, respeitando assim a promoção da Segurança Alimentar e Nutricional (STORCK et al., 2013). Além disso, o aproveitamento integral de frutas e hortaliças, como forma de incentivo ao consumo desse grupo de alimentos, é uma prática alimentar saudável e contribui para a promoção da saúde, já que, são alimentos essenciais à nutrição humana por serem fontes de micronutrientes. Esses alimentos são ricos em vitaminas hidrossolúveis (vitamina C e 
vitaminas do complexo B), vitamina A e minerais, sendo ainda consideradas boas fontes de compostos antioxidantes, que auxiliam na prevenção e até no tratamento de doenças crônicas não transmissíveis como câncer, diabetes e doenças cardiovasculares (GEBBERS, 2007; SHAHIDI, 2009).

Outro benefício de destaque oferecido pelo consumo regular de frutas e hortaliças é o aporte de fibras (OLIVEIRA et al., 2009). A fibra alimentar é a parte comestível das plantas que é resistente à digestão e à absorção pelo intestino delgado humano, cuja fermentação completa ou parcial ocorre no intestino grosso (MIRA et al., 2009). São constituídas de polímeros de carboidratos comestíveis, presentes naturalmente nos alimentos na forma em que estes são consumidas e ainda, são classificadas em solúveis e insolúveis (BERNAUD et al., 2013). Vários estudos sobre as fibras foram realizados nos últimos anos e seus inúmeros benefícios para a saúde têm sido evidenciados. A sua utilização é preconizada para o tratamento e/ou para a prevenção de doenças crônicas: diabetes, hiperlipidemias, obesidade, constipação e neoplasias (TOSTE et al., 2015). Também auxilia na redução do peso corporal e ainda atua na melhora do sistema imunológico (BERNAUD et al., 2013).

Diante disso, o estudo foi baseado na busca pelo entendimento da importância de adesão ao aproveitamento integral de alimentos (AIA), e ainda, pesquisar o impacto que isso traz para a saúde da população, principalmente devido as suas propriedades nutricionais. Portanto, o objetivo deste trabalho foi revisar sobre a importância do aproveitamento integral dos alimentos para a saúde dos indivíduos.

\section{METODOLOGIA}

Foi realizado uma revisão bibliográfica, a partir das bases de dados Scielo (Scientific Electronic Library Online), PubMed (U. S. National Library of Medicine), LILACS (Literatura Latino-Americana e do Caribe em Ciências da Saúde) e Google Scholar. Os termos empregados para fazer a busca foram: aproveitamento integral dos alimentos; desperdício dos alimentos; alimentação saudável; segurança alimentar e nutricional. Os mesmos termos foram pesquisados no idioma em inglês.

Como critérios de inclusão foram utilizados artigos publicados nos últimos 10 anos, com idioma português ou inglês, além de textos na íntegra. Foram excluídos trabalhos incompletos e/ou de outro idioma que não fosse sendo o português ou inglês.

\section{DISCUSSÃO TEÓRICA}

\section{Hábitos Alimentares Saudáveis}

Os hábitos alimentares são relacionados à ideia de ingestão alimentar (ingestão energética e de nutrientes), bem como, a percepção sobre a comida em determinado contexto social, o qual possui a necessidade de repetição e uma ligação com o tempo. As atitudes alimentares do indivíduo são resultantes das interações entre o seu estado fisiológico, psicológico e ambiental e, por isto, é preponderante no desenvolvimento e manutenção da obesidade tempo (KLOTZ et al., 2016).

Nas últimas décadas, diversos estudos científicos estão focados em avaliar a relação entre hábitos 
alimentares e saúde, em diferentes faixas etárias. Como resultado identificaram que a adoção de hábitos alimentares saudáveis e uma alimentação equilibrada, ajuda a prevenir não só a desnutrição em todas as suas formas, como também diferentes doenças transmissíveis e degenerativas crônicas, como diabetes, hipercolesterolemia, obesidade, doenças cardiovasculares e câncer (ZARAGOZA, 2019).

Salienta-se ainda, que o ato de comer vai além da questão fisiológica de nutrir-se, pois está associado a valores culturais, afetivos, sensoriais e sociais, que equivalem à riqueza e a complexidade da vida humana em sociedade. Nesse quesito, a alimentação é uma atividade integrada por um compendio de fatores que se perfazem pelo próprio o ato de se alimentar, a disponibilidade de alimentos e os estágios da cadeia de produtiva de alimentos, composto pelos seguintes estágios: produção, extração (técnicas de colheita e atividades pós-colheita), infraestrutura, processamento, distribuição, armazenamento e comercialização, até o consumidor final (RIBEIRO et al., 2017).

É importante ressaltar, que a alimentação é considerada um direito humano, de necessidade básica, a qual possui uma abordagem multifacetada, visto que, abarca fatores culturais, religiosos, sociais, individuais (PEREIRA et al., 2017). Além disso, uma alimentação adequada ratifica o desenvolvimento do estado de saúde do indivíduo, caracterizado por um completo bem-estar físico, mental e social e não apenas a ausência de doença ou enfermidade (FEIO et al., 2015).

A partir de uma iniciativa do Ministério da Saúde, elaborou-se um documento intitulado, como Guia Alimentar para a População Brasileira, no qual, consta os "10 Passos para a Alimentação Saudável", uma estratégia com recomendações para a adoção de uma alimentação benéfica ao organismo, entre elas, dar prioridade aos alimentos in natura ou minimamente processados, evitando os ultraprocessados e desenvolver habilidades culinárias (JAIME et al., 2015).

De acordo com este guia, os alimentos são conceituados como todas as substâncias sólidas e líquidas que, levadas ao tubo digestivo, são degradadas e posteriormente usadas para formar e/ou manter os tecidos do corpo, regular os processos orgânicos e fornecer energia (JAIME et al.,2015). Carvalho et al. (2011), entendem como alimento, os elementos da ordem da natureza associado a uma concepção de corpo orgânico funcional e objeto de modificações nesse corpo, sendo também um elemento de ordem cultural. Neste sentido, os alimentos são fontes de nutrientes, os quais são compostos por carboidratos, proteínas, lipídios, minerais vitaminas, água e fibras alimentares (MEZOMO, 2015).

Destaca-se que, a alimentação saudável é fundamentada a partir de um estilo dietoterápico, baseado nas necessidades individuais ou, a partir da moderação e do controle da ingestão de alimentos energéticos, ricos em sódio, gorduras saturadas e trans, e em contrapartida, impulsionada pelo estímulo ao consumo de alimentos fontes de fibras, vitaminas e minerais, como as frutas, legumes e verduras (KRAEMER et al., 2014; GOMES et al., 2018).

Nota-se ainda, para atingir um equilíbrio na alimentação é de suma importância adotar como base regular os seguintes princípios dietéticos: quantidade, que é a suficiência calórica da dieta alimentar para repor as perdas energéticas do organismo; a qualidade, constituída pela variabilidade de alimentos e o seu teor de nutrientes; a harmonia, sendo a proporcionalidade entre os nutrientes e o valor calórico total; e por 
fim a adequação, alicerçada no respeito à individualidade (LIMA, 2012).

As inadequações no consumo de nutrientes podem alterar o estado nutricional e resultar em diversos riscos para a saúde, já que, a carência dos nutrientes presentes nos alimentos pode ocasionar a desnutrição e o excesso destes, gera a obesidade (ACUÑA et al., 2004; VINHOLES et al., 2009; CARVALHO et al., 2018). Desse modo, uma alimentação saudável e equilibrada é de extrema importância na definição do estado de saúde dos indivíduos, principalmente quando se trata de doenças crônicas na idade adulta (MAHAN et al., 1994; MONTEIRO et al., 2000).

Por outro lado, são necessários dados sobre composições de alimentos para que a população consiga consumir equilibradamente os nutrientes de acordo com a Ingestão Diária Recomendada (IDR), que é a quantidade de nutrientes que deve ser consumida diariamente para atender às necessidades nutricionais da maior parte dos indivíduos e grupos de pessoas de uma população sadia. A orientação sobre hábitos saudáveis realizada por profissionais de saúde constitui uma importante estratégia na promoção da alimentação saudável em todas as faixas etárias (GOMES et al., 2018).

Dessa forma, é fundamental possibilitar uma alimentação sustentável que consiste em utilizar partes de alimentos que normalmente são desprezadas, como cascas, talos, sementes, entrecascas e folhas amplificando as alternativas para se atingir uma alimentação saudável. O conceito do não desperdício e do aproveitamento integral dos alimentos pode e deve ser realizado no dia a dia por qualquer pessoa, independentemente de sua classe social ou econômica, utilizando produtos menos processados ou ultra processados, valorizando os produtos regionais e a culinária tradicional (GONDIM et al., 2005).

\section{Aproveitamento Integral Dos Alimentos}

De acordo com os dados publicados pela Organização das Nações Unidas, em meados de 2017 metade da população mundial era de aproximadamente 7,6 bilhões de pessoas, e a cada ano, ocorre um aumento de 83 milhões a nível mundial. Mesmo com os índices de natalidade em decréscimo, estima-se que, em 2030, a população global esteja entre 8,4 e 8,6 bilhões de habitantes, e em 2050, entre 9,4 e 10,2 bilhões. Neste contexto, o Brasil é o quinto país mais populoso do mundo, correspondendo a 209 milhões de pessoas, em 2017 (SANTOS et al., 2020).

De acordo com Santos et al. (2020), entre os mais graves efeitos deste aumento populacional, está a exploração dos recursos naturais, onde destaca-se a diminuição de fontes de recursos não renováveis, a perda da biodiversidade e as mudanças climáticas. Além disso, o desperdício de alimentos e os elevados montantes de resíduos gerados a partir destas perdas são temas atuais de grande preocupação e mobilização mundial.

É importante frisar que no mundo são produzidos cerca de 3,9 bilhões de toneladas de alimentos por ano, sendo que destes, 600 milhões de toneladas de produtos industrializados e 260 milhões de toneladas de produtos agropecuários são admitidos pelo Brasil, essa produção equivale a diferentes alimentos, entre eles, carnes, frutas, hortaliças, cereais (FAO, 2018). Nota-se ainda, que o desperdício é maior em países desenvolvidos, os quais possuem tecnologia agrícola e infraestrutura menos desenvolvidas (BELIK et al., 
2012).

Em torno de $25 \%$ desse desperdício é suficiente para erradicar a pobreza, combatendo a insegurança alimentar e nutricional da população, pois a indisponibilidade ao acesso de alimentos, afeta inúmeras famílias (CARVALHO et al., 2018; SANTOS et al., 2020). Estima-se que haverá um aumento para aproximadamente 2,2 bilhões de toneladas/ano, até o ano de 2025 (RIBEIRO et al., 2017)

De acordo com dados da Organização das Nações Unidas para a Agricultura e a Alimentação, a partir do novo relatório, a fome afetou cerca de 42,5 milhões de pessoas, na América Latina e no Caribe (FAO, 2018). A fome é classificada em fome aguda, a qual é momentânea e refere-se à urgência em se alimentar, e a fome crônica, que é permanente, ocorrendo quando a alimentação diária não atinge as necessidades energéticas do indivíduo para a manutenção das atividades essenciais (SILVA et al., 2016).

Sob o mesmo ponto de vista, a pobreza, que tem uma fenomenologia complexa e multidimensional, a qual impossibilita uma parcela representativa da população mundial ao acesso a alimentação, acarreta consequências danosas nos parâmetros físicos, psicológicos e sociais aos indivíduos. No caso do Brasil, para combater à fome, é de vital importância erradicar a pobreza extrema, associada direta ou indiretamente a diversas doenças (ROSANELI et al., 2015).

Verifica-se ainda que, no Brasil a fome e o desperdício de alimentos possuem uma relação não harmônica, pois temos uma parcela significativa de indivíduos sem acesso ao alimento em quantidade e qualidade suficiente, impactando diretamente na segurança alimentar da população (SAATH et al., 2018). O país produz mais de 140 milhões de toneladas de alimentos por ano, um dos maiores exportadores mundiais de alimentos, contudo é um dos campeões de desperdício, demonstrando uma relação paradoxal (KLOTZ et al., 2016).

O Brasil está entre os 10 países que mais perdem alimentos no mundo, onde cerca de $35 \%$ da produção está sendo desperdiçada todos os anos (SCIALABBA, 2015). A plataforma online do Governo do Estado do Rio Grande do Sul, contextualiza essas informações, e destaca que, na Central de Abastecimento do Rio Grande do Sul (CEASA-RS), são geradas 38 toneladas de resíduos orgânicos por dia, sendo equivalente a uma produção diária de uma cidade de 50 mil habitantes (FAO, 2013).

A alimentação alternativa, ou seja, o aproveitamento integral dos alimentos teve início no Brasil a partir do início da década de 80, principalmente para grupos considerados vulneráveis, sendo uma estratégia de combate à fome e desnutrição, pois utiliza alimentos tradicionais e não tradicionais, ricos em vitaminas e minerais, que são acessíveis a toda população (SANTOS et al., 2020).

Os prejuízos econômicos gerados pelo desperdício de alimentos, giram em torno de US\$ 700 bilhões do ponto de vista ambiental e US\$ 900 bilhões na dimensão social (FAO, 2018). Se considerar o tripé da sustentabilidade, sendo ele, econômico, social e ambiental, o custo total relacionado ao desperdício de alimentos situa-se em torno de US\$2,6 trilhões por ano, esse valor corresponde ao PIB do Reino Unido, que atualmente representa a quinta maior economia do mundo (SANTOS et al., 2020). O desperdício é uma questão ética na qual toda sociedade deveria se preocupar (GALIAN et al., 2016).

Analogamente Silvério et al. (2014), ressaltam que no Brasil o desperdício de alimentos representa 
cerca de 12 bilhões de reais por ano. Várias estimativas de desperdício, cito-as: 15\% do desperdício de alimentos ocorre em restaurantes e $20 \%$ em domicílios; e ainda, 39 toneladas de resíduos alimentares são desperdiçadas diariamente, e por fim, 60\% do lixo urbano é de origem alimentar (STORCK et al., 2013; HENZ et al., 2017). Quando se trata de desperdício doméstico, percebe-se que as famílias brasileiras descartam quantidades consideráveis de alimentos devido a fatores culturais, acabam cozinhando além do necessário e grandes porções de alimentos (HENZ et al., 2017). Uma família brasileira com cinco pessoas gasta, em média, $\mathrm{R} \$ 1.532,50$ mensalmente com alimentação e, ao considerar a média mundial de 30\% de desperdício, evidencia que, deste valor, $R \$ 459,75$ são gastos com alimentos que viraram lixo. Ou seja, quase $R \$ 500,00$ da renda familiar foram perdidos (RODRIGUES, 2018).

As perdas são subdivididas em três etapas do processo de produtivo: no campo, no nível do atacado e do varejo (comercialização) e a nível domiciliar. Há cinco categorias de desperdício domiciliar de alimentos, quais sejam, compra excessiva, preparação abundante, cuidado para um animal de estimação, sobras de evitação e preservação inadequada de alimentos (BELIK et al., 2012; HENZ et al., 2017).

Verifica-se ainda que, em nota técnica a Empresa Brasileira de Pesquisa Agropecuária (EMBRAPA), demostra que as perdas pós-colheita de frutos e hortaliças são alarmantes, pautando nos seguintes dados: $10 \%$ dessas perdas acontecem no campo, $50 \%$ no manuseio e transporte, $30 \%$ nas centrais de abastecimento e comercialização e $10 \%$ nos supermercados e consumo doméstico, sendo ineficaz aumentar a produção agrícola do país e não atuar no combate ao desperdício (LIMA, 2012; SOARES, 2013; FERNANDES et al., 2016).

Percebe-se que essa quantidade de alimentos desperdiçados é suficiente atender $78 \%$ dos cinquentas milhões de pessoas que ainda passam fome no país, com 3 refeições diárias. Todavia o desperdício encontra outro impasse na atualidade, haja vista que, o decreto lei no 2.848 , do ano de 1940, obsta que as sobras alimentares sejam doadas, sendo o fornecedor a principal responsável por posteriores complicações relativas ao consumo do alimento doado (SILVÉRIO et al., 2014).

Acrescenta-se a necessidade de mudanças nos padrões de consumo, tendo em vista o imenso desperdício. Dessa maneira, o Aproveitamento Integral dos Alimentos consiste na utilização de um determinado alimento em sua totalidade, como as cascas, sementes, talos e folhas, sendo um procedimento de baixo custo que gera redução do desperdício (GONDIM et al., 2005). Neste sentido, uma alimentação adequada promove a ingestão suficiente dos nutrientes essenciais à sobrevivência, sendo que, o teor de nutrientes das cascas ou talos, em muitos casos, é maior quando comparado à polpa de alguns alimentos, além disso, aproveitar os alimentos na sua forma integral resulta em uma dieta variada, pois aumentam as possibilidades de preparações culinárias (SILVA et al., 2016).

Assim grande parte dos nutrientes que se encontram nas cascas, folhas, talos e sementes de frutas, legumes e verduras, são usualmente descartadas pela maioria da população, cuja justificativa é embasada no hábito inadequado e o mero desconhecimento acerca do aproveitamento dos alimentos, gerando assim desperdício (SILVA et al., 2016).

Essas partes não convencionais dos alimentos podem ser utilizadas para o enriquecimento alimentar, em virtude de possuírem concentrações variadas de nutrientes, consideradas como complementos 
nutricionais, como exemplo, as folhas verdes da couve-flor, que contêm mais ferro que a couve-manteiga, e são mais nutritivas que a própria couve-flor, além disso, tornam as preparações mais palatáveis, dando-lhes mais sabor (SOUZA et al., 2007; FRANZOSI et al., 2018).

A utilização do alimento, de forma sustentável, ou seja, o consumo integral acarreta inúmeros benefícios, tanto para a população quanto na esfera econômica, visto que, atua na redução dos custos de produção, na redução da produção de lixo orgânico, contribuindo para a limpeza dos recursos naturais, prolonga a vida útil do alimento, promove a segurança alimentar e beneficia a renda familiar (SILVA et al., 2009; FRANZOSI et al., 2018).

Com o reaproveitamento das partes não convencionais dos alimentos, esses alimentos que eram destinados aos lixões, são aproveitados para fins nutricionais, reduzindo assim possíveis carências da população. Por isso que hoje a educação nutricional se torna indispensável, para que as pessoas tenham conhecimento de como escolher os alimentos e produzi-los de forma adequada, saudável e segura. Assim formando hábitos alimentares mais saudáveis, promovendo uma melhor qualidade de vida e com isso reduzindo o desperdício de alimentos (LAURINDO et al., 2014; PEREIRA et al., 2017).

Outro ponto importante, está na ligação entre o desperdício de alimentos e o impacto negativo no meio ambiente, em função da inadequada deposição do lixo alimentar no solo, tendo consequências como o odor gerado pela putrefação da matéria orgânica e a formação do chorume que normalmente encontra-se contaminado e tem potencial para atingir rios e os lençóis freáticos (SANTOS et al., 2020).

Diante do quadro social e econômico da população brasileira, o estudo da utilização integral de hortaliças no uso doméstico, bem como sua incorporação na elaboração de produtos industrializados, pode contribuir substancialmente para aumentar a disponibilidade de nutrientes, sendo uma fonte de baixo custo de proteínas, fibras, vitaminas e minerais (PEREIRA et al., 2017).

No que tange ao aporte de fibras nos alimentos, percebe-se que são classificadas em: fibras solúveis, as que reduzem o tempo de trânsito intestinal e ajudam na diminuição das concentrações séricas de colesterol, além disso, melhoram a tolerância à glicose, e atua na melhora de problemas cardiovasculares, e por fim as fibras insolúveis, que apesar de não terem ação na colesterolemia, atuam no aumento da saciedade e auxiliam na redução da ingestão energética, com consequente promoção da perda de peso (MIRA et al., 2009).

Portanto evitando o desperdício, é possível diminuir em até 30\% os gastos com alimentação. Além disso, aproveitar os alimentos ao máximo é uma maneira de colaborar para a diminuição efetiva do lixo orgânico, que representa cerca de 65\% de todo o lixo produzido no país (MORETTI, 2003).

Com a redução do desperdício e perda de alimentos, não só evitaria a pressão sobre recursos naturais escassos, nas mudanças climáticas (emissões de gases de efeito estufa), mas também diminuiria a necessidade de aumentar a produção de alimentos, portanto a FAO propôs ações como, o incentivo ao consumo de frutas, hortaliças e verduras "feias" ou amassadas, pesquisas sobre técnicas de manejo e póscolheita, aumento da validade dos produtos, embalagens inteligentes, desenvolvimento de alimentos funcionais e de educação alimentar como o consumo de cascas de banana e folhas de couve flor, por exemplo 
(CARVALHO et al., 2018).

\section{Programas que incentivam o aproveitamento integral dos alimentos}

Um documento publicado pelo programa das Nações Unidas para o Meio Ambiente, destaca que, antes de definir qualquer estratégia para prevenir ou reduzir o desperdício de alimentos, é importante entender como eles são gerados em escala nacional e regional. A partir dos resultados, dar prioridade as áreas de ação e mensurar as mudanças conquistadas a partir delas, incluindo, tanto as perdas e os desperdícios que surgem na cadeira de suprimento de alimentos e bebidas quando os gerados pelos consumidores dentro e fora de suas residências (SANTOS et al., 2020).

Para que as informações possam se comunicar de forma eficiente, estas devem envolver, produtores de alimentos, estabelecimentos agroindustriais, distribuidores e comerciários (atacado e varejo, os quais integram a cadeira produtiva), administrações, órgãos e demais autoridades públicas e também quem consome. Esta relação ocorre para que sejam reduzidas as disparidades relacionadas com o volume de oferta e demanda, em diversas situações como: a) quando apodrece parte do que foi produzido no campo, devido ao produtor não encontrar compradores ou preços que cubram os custos da produção, b) quando um indivíduos prepara uma refeição para uma quantidade de pessoas, mas apenas metade comparecem, c) quando um restaurante planeja de forma incorreta a demanda e estoca produtos além do necessário, tento posteriormente que descarta-los (FAO, 2013).

O Brasil é hoje referência internacional quando se trata de políticas de segurança alimentar (GRAZIANO et al., 2010). Mas, apesar da abundância de terras produtivas, da grandiosidade territorial e da larga escala de produção de alimentos, o Brasil ainda luta para combater a fome. O problema não é a falta de comida, mas a perda e o desperdício de alimentos, tanto ao longo das cadeias produtivas e de distribuição quanto no preparo e armazenamento (FONTES et al., 2015).

Os programas de redistribuição ou doação de alimentos são métodos eficientes para reduzir a perda e o desperdício de alimentos. O Programa do SESI - Alimente-se Bem/Cozinha Brasil foi inaugurado em meados de 1999. A ideia surgiu após uma pesquisa alimentar com 1.600 trabalhadores de indústrias de pequeno e médio porte na região norte da capital de São Paulo, a qual apurou que $62 \%$ desses trabalhadores se alimentavam erroneamente, com um consumo mínimo de verduras, frutas e legumes. Outro fator preponderante para a implantação do programa foi a constatação de que, no Brasil, alimentos de grande valor nutritivo normalmente são transformados em ração animal ou vão direto para o lixo. A partir dessas conclusões, o Serviço Social da Indústria de São Paulo (Sesi-SP) criou o Alimentasse Bem, cujo intuito é ensinar a população a se alimentar melhor, gastando menos e evitando o desperdício. O programa leva conhecimento ao trabalhador e à sua família, oferecendo-lhes a oportunidade de elaborar refeições saudáveis e de baixo custo. Isso é possível porque nas preparações recomendadas pelo programa os alimentos são utilizados integralmente, sem o desperdício de cascas, talos e folhas de legumes e frutas, e uma variedade de pratos pode ser elaborada a partir de um único produto (FONTES et al., 2015).

O Mesa Brasil SESC é um Programa de Segurança Alimentar e Nutricional voltado para a inclusão 
social, constituindo-se numa rede Nacional de Solidariedade contra a fome e o desperdício. O Programa tem caráter permanente e as ações vão além da arrecadação e distribuição de alimentos doados. São desenvolvidos, em parceria com o Programa Alimentos Seguros (PAS) e o SENAC, materiais e capacitações para produção de uma alimentação segura, saudável e saborosa conforme padrões estabelecidos pela Vigilância Sanitária, bem como para utilização do alimento de forma integral. A série "MESA BRASIL SESC Segurança Alimentar e Nutricional" - é um conjunto de materiais educativos desenvolvidos pelo SESC, SENAC e PAS - Programa Alimentos Seguros, como instrumento para garantir a qualidade e o aproveitamento integral dos alimentos - desde a doação, na empresa parceira, até o consumo final, na entidade beneficiada pelo Programa. Os materiais desta série fazem parte das ações estruturais do MESA BRASIL SESC (relacionadas às Políticas Específicas do Programa Fome Zero do Governo Federal), que objetivam contribuir para a inclusão nutricional e social de segmentos da população em situação de vulnerabilidade alimentar, através de uma ação educativa intensa voltada à Educação Alimentar, difusão de conhecimentos e promoção da cidadania (SANTOS et al., 2020).

Outro programa brasileiro é chamado de A Fruta Imperfeita, sendo uma inciativa que busca a propagação do consumo consciente, atuando como promotor da conexão entre os fornecedores e os usuários. Os alimentos que antes seriam descartados pelos pequenos produtores, são comprados e revendidos em forma de cestas que possuem 14 variedades de frutas, legumes e verduras. Os consumidores podem fazem a aquisição dos produtos de forma avulsa ou ainda através do formato de assinatura semanal ou quinzenal. Com essa inciativa, já foram salvas, mais de 300 toneladas em apenas dois anos de atividades (SANTOS et al., 2020).

A Nestlé criou o Programa Nutrir, cujo principal objetivo é prevenir a desnutrição em crianças com idades entre 5 e 14 anos. A motivação do projeto foi à percepção de que muitos casos de desnutrição não ocorrem necessariamente devido à falta de alimento, mas por causa das opções sobre o que se vai comer ou pela forma de utilizar o alimento disponível. Preferir alimentos industrializados ao invés de frutas e verduras frescas, ou usar somente o suco da laranja e não a fruta inteira são escolhas importantes que interferem na qualidade da alimentação e na saúde de qualquer pessoa. Quando se trata de crianças que vivem em condições socioeconômicas desfavoráveis, o problema torna-se mais grave. Tudo começou com a ideia de ensinar conceitos de alimentação saudável para mães e crianças, mas sempre por meio de brincadeiras e receitas que incentivam o aproveitamento integral dos alimentos (MARIUZZO, 2007).

O Banco de Alimentos é definido formalmente pelo Ministério do Desenvolvimento Social e Combate à Fome (MDS) como "uma iniciativa de abastecimento e segurança alimentar que tem como objetivo a redução do desperdício de alimentos, o aproveitamento integral dos alimentos e a promoção de hábitos alimentares saudáveis, contribuindo diretamente para a diminuição da fome de populações vulneráveis, assistidas ou não por entidades assistenciais". Já a Associação Canadense de Bancos de Alimentos define o Banco de Alimentos como "uma estrutura logística e uma organização sem fins lucrativos com o objetivo de coletar, armazenar e distribuir comida, sem custos, diretamente ou através de agências que também podem fornecer refeições para pessoas com fome" (GONÇALVES et al., 2011). 
De fato, na definição conceitual de Bancos de Alimentos, o aspecto da estrutura logística é fundamental para distingui-los das iniciativas de colheita urbana, que visam ao mesmo objetivo (distribuição alimentar e combate ao desperdício), mas atuam apenas por meio de coleta e distribuição imediata, não requerendo infraestrutura específica de beneficiamento nem área para estocagem. Os Bancos de Alimentos, neste sentido, são organizações que contam com estrutura logística capaz de receber, selecionar, avaliar, selecionar e doar alimentos para instituições e pessoas necessitadas, podendo ter múltiplos propósitos de ação comunitária, como orientação nutricional, orientações jurídicas, distribuição de outros gêneros de consumo (BELIK et al., 2012; SANTOS et al., 2020).

O Programa Nacional de Alimentação e Nutrição (PRONAN) representou, pela primeira vez, a oportunidade do Estado formalizar uma política oficial para a alimentação e nutrição, com abrangência de nove programas: (a) Programa de Nutrição em Saúde; (b) Programa de Alimentos Básicos em Área de Baixa Renda; (c) Programa de Compra Direta do Pequeno Produtor; (d) Programa Nacional de Incentivo ao Aleitamento Materno; (e) Programa de Combate as Carências Especificas; (f) Sistema de Vigilância Alimentar e Nutricional; (g) Programa Nacional de Alimentação Escolar; (h) Programa de Alimentação ao Trabalhador; (i) Programa de Alimentação Popular (ALBUQUERQUE, 2006)

Outros projetos também visam à educação alimentar e o combate ao desperdício, como a Arno (Aprendendo na Prática) onde talos de beterraba e casca de banana são apenas alguns dos ingredientes das receitas ensinadas pelo Aprendendo na Prática. Em 2004, 1.100 entidades sociais participaram do "Parcerias Contra o Desperdício". Além disso, auxiliares de cozinha e nutricionistas de 175 entidades foram capacitados pelo programa para melhor aproveitamento dos alimentos e receberam noções de segurança alimentar e nutrição. Em parceria com o programa "Banco de Alimentos", do SESC, e o projeto "Cozinha Brasil", do Sesi (baseado no programa Alimente-se Bem), cerca de 21 mil pessoas foram beneficiadas direta e indiretamente pela capacitação de 4.375 pessoas (GONÇALVES et al., 2005).

A Associação de Apoio a Políticas de Segurança Alimentar foi criada para estimular a participação do meio empresarial no Programa Fome Zero do governo federal. A Apoio Fome Zero é uma organização da sociedade civil de interesse público formada por empresas e entidades empresariais, unidas com o propósito de amparar e estimular ações e projetos que promovam a segurança alimentar e nutricional da população brasileira. Seu principal objetivo é criar uma cultura da solidariedade entre empresários, demonstrando que a busca de bons resultados na operação de empresas pode ser combinada com a melhora das condições sociais do país (MORETTI, 2003).

O projeto Ripasa - Cidadania na Mesavisa reduzir em no mínimo $30 \%$ a quantidade de alimentos desperdiçados e transformar esse montante em doações para a comunidade carente, ou seja, o que se consegue reduzir é doado. Para isso, o programa conta com duas ferramentas que ajudam a conscientizar e estimular os cidadãos. Uma delas é o curso de reeducação alimentar, oferecido pelo SESI que chama a atenção para os hábitos alimentares e a conduta na redução das sobras. A outra é o referencial social, porque a quantidade de alimentos não desperdiçada é revertida em mantimentos in natura para organizações sociais que trabalham com crianças, adolescentes, adultos e famílias de baixa renda (GONÇALVES et al., 2005). 
A Food and Agriculture Organization of the United Nations (2018) alerta que a conscientização é o primeiro passo para a prevenção e deve-se iniciar campanhas educativas voltadas para o consumo. Visando isso, a Save Food Brasil, uma inciativa brasileira vinculada a FAO, busca estimular a união, cooperação e a intercomunicação entre grupos e corporações com o objetivo de promover a redução das perdas do desperdício de alimentos no Brasil. Seus objetivos principais são: construir uma rede de especialistas brasileiros na área, estimular e simplificar a comunicação entre setores, manter a rede atualizada, e buscar a sensibilização da sociedade sobre a relevância do assunto (SANTOS et al., 2020)

A grande relevância que o tema de desperdício de alimentos, abrangeu a nível mundial, levou diversos países a assumirem compromissos em marcos estratégicos internacionais, como o Acordo de Paris, firmado na 21ạ. Conferência das Partes da Convenção - Quadro das Nações Unidas sobre Mudança do Clima, a Agenda 2030 e o Plano da Comunidade de Estados Latino-americanos e Caribenhos para a Segurança Alimentar, Nutricional e Erradicação da Fome 2025. É proposta uma atuação a nível mundial, promovida pelos governos, empresas, instituições de ensino e a sociedade civil, para atingir 17 objetivos e suas respectivas 169 metas, que possibilitem a erradicação da pobreza e proporcionem uma vida dignada para todos (SANTOS et al., 2020).

\section{CONCLUSÕES}

O desenvolvimento do presente estudo possibilitou uma análise de que com o reaproveitamento das partes não convencionais dos alimentos que são as partes desprezadas dos alimentos como as cascas, talos e folhas, além de oferecer uma refeição mais saudável, devido às altas concentrações de propriedades nutricionais como vitaminas, minerais e fibras comparadas às partes convencionais dos alimentos, proporcionaria diversos benefícios.

Dentre esses benefícios pode-se destacar a prevenção de doenças crônicas não transmissíveis, melhora da qualidade de vida, redução de peso corporal, melhora do sistema imunológico, redução dos gastos com a alimentação, além do mais, o impacto ambiental que traz é significante, pois reduziria o desperdício e o lixo orgânico gerado, reduzindo o desperdício de alimentos e consequentemente reduzindo a fome. Portanto, a importância da educação sobre a as partes não convencionais para a população é de suma importância para que tenham o conhecimento desse assunto, possibilitando a população de uma alimentação mais nutritiva, saborosa e sustentável.

\section{REFERÊNCIAS}

ACUÑA, K.; CRUZ, T.. Avaliação do estado nutricional de adultos e idosos e situação nutricional da população brasileira. Arquivos Brasileiros de Endocrinologia \& Metabologia, v.48, n.3, p.345-361, 2004. DOI: http://doi.org/10.1590/S0004-27302004000300004

ALBUQUERQUE, M. D. C.. Participação popular em políticas públicas: espaço de construção da democracia brasileira. São Paulo: Instituto Pólis, 2006.

BELIK, W. B.; CUNHA, A. R. A. A.; COSTA, L. A.. Crise dos alimentos e estratégias para a redução do desperdício no contexto de uma política de segurança alimentar e nutricional no Brasil. Planejamento e Políticas Públicas, n.38, 2012

BERNAUD, F. S. R.; RODRIGUES, T. D. C.. Fibra alimentar: ingestão adequada e efeitos sobre a saúde do metabolismo. Arquivos Brasileiros de Endocrinologia \& Metabologia, v.57, p.397-405, 2013. DOI: http://doi.org/10.1590/S0004-27302013000600001 
BRASIL. Ministério da Saúde. Secretaria de Atenção à Saúde. Departamento de Atenção Básica. Política nacional de alimentação e nutrição. Brasília: MS, 2008.

CARVALHO, L. C. C.; LEDUC, E.; AMORIM, A.; HELBINGEN, A. B.; BRANCO, C.; LOURENÇO, L.; LOPES, M. A.. Desafio 2050 e os objetivos de desenvolvimento sustentável. AgroANALYSIS, v.38, p.31-38, 2018.

CARVALHO, M. C. D. V. S.; LUZ, M. T.; PRADO, S. D.. Comer, alimentar e nutrir: categorias analíticas instrumentais no campo da pesquisa científica. Ciência \& Saúde Coletiva, v.16, p.155-163, 2011. DOI: http://doi.org/10.1590/S1413-81232011000100019

FEIO, A.; OLIVEIRA, C. C.. Conceptual convergences and divergences in health education. Saúde e Sociedade, v.24, p.703-715, 2015. DOI: http://doi.org/10.1590/S010412902015000200024

FERNANDES, C. C.; MAZZOLA, B. G.; JÚNIOR, M. D. M. O.. Resíduos alimentares e as mudanças climáticas. Organizações e Sustentabilidade, v.4, p.116-141, 2016. DOI: http://doi.org/10.5433/23189223.2016v4n2p116

FILIMONAU, V.; TODOROVA, E.; MZEMBE, A.; SAUER, L.; YANKHOLMES, A.. A comparative study of food waste management in full service restaurants of the United Kingdom and the Netherlands. Journal of Cleaner Production, p.120775, 2020. DOI: http://doi.org/10.1016/j.jclepro.2020.120775

FONTES, M. B.; CRIVELARO, R. C.; AMARAL, R. S.; FERREIRA, G. M.; PERES, A. P.. Avaliação dos Impactos Econômicos e Sociais do Programa Cozinha Brasil (Período 20092010). Revista Meta: Avaliação, v.7, p.232-264, 2015.

FAO. Food and Agriculture Organization of the United Nations. Retail food waste action guide. FAO, 2018.

FAO. Food and Agriculture Organization of the United Nations. Food wastage footprint: Impacts on natural resources. FAO, 2013.

FRANZOSI, D.; DANELUZ, H. C.; BARATTO, I.. Níveis de desperdício de partes não convencionais de produtos utilizados diariamente em um restaurante no sudoeste do Paraná. Revista Brasileira De Obesidade, Nutrição e Emagrecimento, v.12, p.66-75, 2018.

GALIAN, L.; SANTOS, S. S.; MADRONA, G. S.. Análise do desperdício de alimentos em uma unidade de alimentação e nutrição. Revista GEINTEC-Gestão, Inovação e Tecnologias, v.6, p.3121-3127, 2016. DOI: http://doi.org/10.7198/S2237-0722220160002010

GEBBERS, J. O.. Atherosclerosis, cholesterol, nutrition, and statins: a critical review. GMS German Medical Science, v.5, 2007.

GOMES, A. P.; BIERHALS, I. O.; VIEIRA, L. S.; SOARES, A. L. G.; FLORES, T. R.; ASSUNÇÃO, M. C. F.; GONÇALVES, H.. Dietary patterns of elderly Brazilians and associated determinants: a population-based study in the south of Brazil. Ciência \& Saúde Coletiva, v.25, p.1999-2008, 2020. DOI: http://doi.org/10.1590/1413-81232020256.20932018
GONÇALVES, B. S.; MAGRI, C.; FORLENZA, K. B.; SILVA, L. F.; MIRRA, M.; ITACARAMBI, P.. O compromisso das empresas com o combate ao desperdício de alimentos: Banco de alimentos, colheita urbana e outras ações. São Paulo: Instituto Ethos, 2005.

GONÇALVES, M. P.; CAMPOS, S. T. D.; SARTI, F. M.. Políticas públicas de segurança alimentar no Brasil: uma análise do Programa de Restaurantes Populares. Revista Gestão \& Políticas Públicas, v.1, n.1, p.92-111, 2011.

GONDIM, J. A. M.; MOURA, M. D. F. V.; DANTAS, A. S.; MEDEIROS, R. L. S.; SANTOS, K. M.. Composição centesimal e de minerais em cascas de frutas. Food Science and Technology, v.25, n.4, p.825-827, 2005. DOI: http://doi.org/10.1590/S0101-20612005000400032

GRAZIANO, J.; DEL GROSSI, M. E.; FRANÇA, C. G.. Fome Zero: a experiência brasileira. Brasília: Ministério do Desenvolvimento Agrário, 2010.

HENZ, G. P.; PORPINO, G.. Food losses and waste: how Brazil is facing this global challenge? Horticultura Brasileira, v.35, n.4, p.472-482, 2017. DOI: http://doi.org/10.1590/s0102053620170402

JAIME, P. C.; STOPA, S. R.; OLIVEIRA, T. P.; VIEIRA, M. L.; SZWARCWALD, C. L.; MALTA, D. C.. Prevalência e distribuição sociodemográfica de marcadores de alimentação saudável, Pesquisa Nacional de Saúde, Brasil 2013. Epidemiologia e Serviços de Saúde, v.24, p.267-276, 2015. DOI: http://doi.org/10.5123/S1679-49742015000200009

KLOTZ-SILVA, J.; PRADO, S. D.; SEIXAS, C. M.. Comportamento alimentar no campo da Alimentação e Nutrição: do que estamos falando?. Physis: Revista de Saúde Coletiva, v.26, p.1103-1123, 2016. DOI: http://doi.org/10.1590/S0103-73312016000400003

KRAEMER, F. B.; PRADO, S. D.; FERREIRA, F. R.; CARVALHO, M. C. V. S. D.. O discurso sobre a alimentação saudável como estratégia de biopoder. Physis: Revista de Saúde

Coletiva, v.24, p.1337-1360, 2014. DOI: http://doi.org/10.1590/S0103-73312014000400016

LAURINDO, T. R.; RIBEIRO, K. A. R.. Aproveitamento integral de alimentos. Interciência \& Sociedade, v.3, n.2, 2014.

LIMA, G. P. P.; SILVA, J. A. T.; BERNHARD, A. B.; PIROZZI, D. C. Z.; FLEURI, L. F.; VIANELLO, F.. Procedimentos de fertilização orgânica e convencional sobre o teor de nitratos, antioxidantes e pesticidas em partes de vegetais. Aditivos alimentares e contaminantes: Parte B, v.5, n.3, p.188-193, 2012. DOI: http://doi.org//10.1080/19393210.2012.695398

MAHAN, L. K.; ESCOTT-STRUMP, S. K.. Alimentos, nutrição e dietoterapia. São Paulo: Roca, 1994.

MARIUZZO, P.. Programa Nutrir: alimentação saudável e voluntariado. Inovação Uniemp, v.3, n.4, p.48-49, 2007.

MEZOMO, I. D. B.. Os serviços de alimentação: planejamento e administração. São Paulo: Manole 2015.

MIRA, G. S.; GRAF, H.; CÂNDIDO, L. M. B.. Visão retrospectiva em fibras alimentares com ênfase em beta-glucanas no 
tratamento do diabetes. Brazilian Journal of Pharmaceutical Sciences, v.45, n.1, p.11-20, 2009. DOI: http://doi.org/10.1590/S1984-82502009000100003

MONTEIRO, C. A.; MONDINI, L.; COSTA, R. B.. Mudanças na composição e adequação nutricional da dieta familiar nas áreas metropolitanas do Brasil (1988-1996). Revista de Saúde Pública, v.34, n.3, p.251-258, 2000. DOI: http://doi.org/10.1590/S0034-89102000000300007

MORETTI, C.. Pesquisa, Desenvolvimento e Inovação Tecnológica: buscando soluções para a redução do desperdício de alimentos no país. In: Diálogos Akatu: o fome zero e o consumo consciente de alimentos. São Paulo: Instituto Akatu, 2003. p.10-14.

OLIVEIRA, A. C. D.; VALENTIM, I. B.; GOULART, M. O. F.; SILVA, C. A.; BECHARA, E. J. H.; TREVISAN, M. T. S.. Fontes vegetais naturais de antioxidantes. Química Nova, v.32, n.3, p.689-702, 2009. DOI: http://doi.org/10.1590/S010040422009000300013

PEREIRA, I. E.; QUEIROZ, A. D. M.; FIGUEIREDO, R. D. Características físico-químicas do tomate em pó durante o armazenamento. Revista de biologia e ciências da terra, v.6, n.1, p.83-90, 2006

PORPINO, G.; PARENTE, J.; WANSINK, B.. Paradoxo do desperdício de alimentos: antecedentes do descarte de alimentos em domicílios de baixa renda. Revista internacional de estudos do consumidor, v.39, n.6, p.619629, 2015. DOI: http://doi.org/10.1111/ijcs.12207

RIBEIRO, H.; JAIME, P. C.; VENTURA, D.. Alimentação e sustentabilidade. Estudos avançados, v.31, n.89, p.185-198, 2017. DOI: http://doi.org/10.1590/s010340142017.31890016

RODRIGUES, P.. Projeto incentiva consumo consciente de hortaliças para evitar o desperdício nas residências. Hortaliças em Revista: Embrapa Hortaliças, v.6, n.23, p.6-15, 2018.

ROSANELI, C. F.; RIBEIRO, A. L. C.; ASSIS, L. D.; SILVA, T. M. D.; SIQUEIRA, J. E. D.. La fragilidad humana frente a la pobreza y el hambre. Revista Bioética, v.23, n.1, p.89-97, 2015. DOI: http://doi.org/10.1590/1983-80422015231049

SAATH, K. C. D. O.; FACHINELLO, A. L.. Crescimento da demanda mundial de alimentos e restrições do fator terra no Brasil. Revista de Economia e Sociologia Rural, v.56, n.2, p.195-212, 2018. DOI: http://doi.org/10.1590/123456781806-94790560201

SANTOS, K. L. D.; PANIZZON, J.; CENCI, M. M.; GRABOWSKI, G.; JAHNO, V. D.. Perdas e desperdícios de alimentos: reflexões sobre o atual cenário brasileiro. Brazilian Journal of Food Technology, v.23, 2020. DOI:

http://doi.org/10.1590/1981-6723.13419
SCIALABBA, N.. Food wastage footprint \& Climate Change. UN FAO, p.15-19, 2015.

SHAHIDI, F.. Nutraceuticals and functional foods: whole versus processed foods. Trends in Food Science \& Technology, v.20 n.9, p.376-387, 2009. DOI: http://doi.org/10.1016/j.tifs.2008.08.004

SILVA, A. B. D.; WIEST, J. M.; CARVALHO, H. H. C.. Compostos químicos e atividade antioxidante analisados em Hibiscus rosa-sinensis L.(mimo-de-vênus) e Hibiscus syriacus L.(hibisco-da-síria). Brazilian Journal of Food Technology, v.19, p.1-9, 2016. DOI: http://doi.org/10.1590/1981-6723.7415

SILVA, M. B. D. L.; RAMOS, A. M.. Composição química, textura e aceitação sensorial de doces em massa elaborados com polpa de banana e banana integral. Revista Ceres, v.56, n.5, p.551-554, 2009.

SILVÉRIO, G. D. A.; OLTRAMARI, K.. Desperdício de alimentos em Unidades de Alimentação e Nutrição brasileiras. Ambiência, v.10, n.1, p.125-33, 2014. DOI: http://doi.org/10.5935/ambiencia.2014.01.10

SOARES, A. G.. Desperdício de Alimentos no Brasil: um desafio político e social a ser vencido. Embrapa Agroindústria de Alimentos: MAPA, 2014.

SOUZA, P. D.; NOVELLO, D.; ALMEIDA, J. M.; QUINTILIANO, D. A.. Análise sensorial e nutricional de torta salgada elaborada através do aproveitamento alternativo de talos e cascas de hortaliças. Alimentos e Nutrição, v.18, n.1, p.5560, 2007.

STORCK, C. R.; NUNES, G. L.; OLIVEIRA, B. B. D.; BASSO, C. Folhas, talos, cascas e sementes de vegetais: composição nutricional, aproveitamento na alimentação e análise sensorial de preparações. Ciência Rural, v.43, n.3, p.537543, 2013. DOI: http://doi.org/10.1590/S010384782013000300027

TOSTE, C. F.; FRÓES, S. C.; FRIEDE, R.; JUNQUEIRA M. C.; MIRANDA, M. G.; AVELAR, K. E. S.. Aproveitamento integral de Alimentos e o seu impacto na Saúde. Sustentabilidade em Debate, v.6, n.3, p.131-143, 2015. DOI: http://doi.org/10.18472/SustDeb.v6n3.2015.16105

VINHOLES, D. B.; ASSUNÇÃO, M. C. F.; NEUTZLING, M. B.. Frequência de hábitos saudáveis de alimentação medidos a partir dos 10 Passos da Alimentação Saudável do Ministério da Saúde: Pelotas, Rio Grande do Sul, Brasil. Cadernos de Saúde Pública, v.25, p.791-799, 2009.

ZARAGOZA-MARTÍ, A.. Influência da ingestão de alimentos ou grupos de alimentos na ocorrência e/ou proteção de diferentes tipos de câncer: revisão sistemática. Nutricion Hospitalaria, v.37, n.1, p.169-192, 2019. DOI: http://doi.org/10.20960/nh.02588

A CBPC - Companhia Brasileira de Produção Científica (CNPJ: 11.221.422/0001-03) detém os direitos materiais desta publicação. Os direitos referem-se à publicação do trabalho em qualquer parte do mundo, incluindo os direitos às renovações, expansões e disseminações da contribuição, bem como outros direitos subsidiários. Todos os trabalhos publicados eletronicamente poderão posteriormente ser publicados em coletâneas impressas sob coordenação da Sustenere Publishing, da Companhia Brasileira de Produção Científica e seus parceiros autorizados. Os (as) autores (as) preservam os direitos autorais, mas não têm permissão para a publicação da contribuição em outro meio, impresso ou digital, em português ou em tradução. 